\title{
3D digital breast tomosynthesis versus US in evaluating breast asymmetries
}

\author{
Omnia Mokhtar ${ }^{1}$, Sahar Abd elsalam², Gehan Gamal ${ }^{3}$, Eman Naguib ${ }^{4}$ and Manal Gomaa, ${ }^{5,6^{*}}$
}

\begin{abstract}
Background: Digital breast tomosynthesis (DBT) now become one of the available diagnostic imaging modalities of the breast, and the present study was done to evaluate its diagnostic value versus that of breast ultrasound (US) in the evaluation of breast asymmetries. This study included 51 patients with 57 mammography identified breast asymmetries; their ages were ranged from 26 to 72 years (mean age $50.05 \pm 8.1$ SD). For all patients, both digital breast tomosynthesis and ultrasound were done, and their results were compared.

Results: Tomosynthesis in this study showed better diagnostic performance compared to mammography; the sensitivity of tomosynthesis was $83.33 \%$, the specificity was $89.74 \%$, the positive predictive value was $78.95 \%$, the negative predictive value was $92.11 \%$, and the accuracy was $87.71 \%$ while the sensitivity of mammography was $72.22 \%$, the specificity was $71.79 \%$, the positive predictive value was $54.17 \%$, the negative predictive value was 84.85 $\%$, and the accuracy was $71.92 \%$.

Breast ultrasound showed the highest sensitivity in this study with the sensitivity of ultrasound being $100.00 \%$, the specificity being $92.31 \%$, the positive predictive value being $85.71 \%$, the negative predictive value being $100.00 \%$, and the accuracy being $94.73 \%$.

Conclusion: Tomosynthesis enables better depiction of asymmetries. It can be useful in the screening setting where better lesion detection and accurate description of lesions is desired. Therefore, it can detect more cancers and can reduce the number of biopsies.

Breast ultrasound should be coupled with breast mammography and 3D tomosynthesis in the evaluation of the breast asymmetries as it reduces false-negative results, detects solid and cystic lesions, and assesses solid lesion nature.
\end{abstract}

Keywords: Digital breast tomosynthesis, Ultrasound, Mammography, Breast asymmetries

\section{Background}

Although there is clearly a wide variation in breast size and parenchymal pattern, the breasts have fairly symmetric internal structures with similar densities and architectures at mammography, while asymmetric breast densities are relatively frequently seen at screening mammography [1].

Asymmetric breast tissue is usually benign and secondary to variations in normal breast tissue, postoperative

\footnotetext{
* Correspondence: mibraheim3333@gmail.com

${ }^{5}$ Department of Diagnostic and Interventional Radiology, National Liver Institute, Menofyia University, Al Minufya, Egypt

${ }^{6}$ Al-Haram Al-, Giza, Egypt

Full list of author information is available at the end of the article
}

change, or hormone replacement therapy. However, an asymmetric area may indicate a developing mass or an underlying cancer [2].

There are different types of asymmetries. If a finding is seen originally on only one standard screening view, it is called Single-View Asymmetry [3]. A Global asymmetry is an asymmetry which involves a greater volume of breast tissue [1]. A focal asymmetry is a focal mammographic abnormality visible on at least two different mammographic projections [3]. A developing asymmetry is a focal asymmetry that is new or increasing in size or conspicuity compared with prior studies [3]. 
Breast cancer risk in a developing asymmetry is sufficient to recommend biopsy (Breast Imaging Reporting and Data System) even in the absence of a sonographic correlate [3]. Breast ultrasonography (US) has long been used as a popular problem-solving diagnostic tool, as it is widely available and well-tolerated by patients [4].

Digital breast tomosynthesis is an advanced technique that offers a potential advantage for evaluation of masses, areas of architectural distortion, and asymmetries compared with conventional two-dimensional mammographic images [5].

The aim of the work is to evaluate the diagnostic value of digital breast tomosynthesis (DBT) versus US in the evaluation of breast asymmetries regarding the sensitivity, specificity, positive predictive value, negative predictive value, and accuracy.

\section{Methods}

This prospective study was conducted at the radiology department and included 51 patients who performed diagnostic or screening mammograms during the period from August 2015 to February 2016. Their mammograms showed breast asymmetries, which were unilateral in 44 cases and bilateral in 7 cases. Their ages ranged from 26 to 72 years (mean age $50.05 \pm 8.1 \mathrm{SD}$ ). Mammography was done for young patients because they had high-risk family history and complaints of lump-like lesion, so exclusion of microcalcifications by mammography was important for them.

Inclusion criteria were patients with only breast asymmetries in mammography.

Exclusion criteria were contraindication to mammography, e.g., pregnant women and in patients presenting with mass lesions or only calcifications.

All patients were subjected to the following:

1. Demographic and clinical data collection: Including patient's name, age, marital status and number of offspring, residence, phone number, diagnosis, duration of illness, past history, and family history.

2. Imaging procedure: All patients underwent digital mammography, 3D tomosynthesis (mediolateraloblique (MLO) and cranial-caudal (CC) views), and breast ultrasound.

\section{Equipment}

1. Mammographic examination was performed using the Senographe Essential, GE healthcare Full-Field Digital Mammography machine that was upgraded to provide tomosynthesis. Senographe Essential has dual anode (rhodium molybdenum) with CsI digital detector.
2. The ultrasound examination was performed using LOGIQ ${ }^{\mathrm{m}} \mathrm{P} 5$ ultrasound scanner (GE Health care) linear array electronic probe.

\section{Technique of full-field digital mammography}

Standard views, mediolateral-oblique and craniocaudal, were taken for all patients.

\section{Technique of 3D tomosynthesis}

For 3D digital tomosynthesis, two views (MLO and CC) were obtained. 3D DBT involved the acquisition of 12 to $152 \mathrm{D}$ projection exposures by a digital detector from a mammographic $\mathrm{x}$-ray source which moves over a limited arc angle. The 3D volume of compressed breast was reconstructed from the 2D projections in the form of series of images (slices) through the entire breast. Images were assessed in the workstation.

\section{Technique of breast ultrasound}

Radial scanning of the entire breast and axillary tail of both sides was performed with the patient's arm relaxed and flexed behind the head. Medial lesions were scanned in the supine position, and lateral lesions, including the axilla, were scanned with the patient in the contralateral oblique position. Longitudinal and transverse images of breast lesions were obtained.

\section{Image analysis and interpretation of mammography and tomosynthesis}

Image interpretation was done by two radiologists, one of them with more than 20 years of experience and the other with more than 10 years of experience in mammography. Breast density was assessed for each patient. Each asymmetry was evaluated regarding site and type of asymmetry (one view, focal, global asymmetry). The Breast Imaging Reporting and Data System (BIRADS) category of each lesion was determined according to the BIRADS atlas 2013 guided by the results of clinical data and mammographic findings but blind to final pathologic diagnosis. Tomosynthesis was also interpreted by the radiologists in the same way as mammography

Image analysis and interpretation of breast ultrasound Each lesion was evaluated regarding shape, boundary, margin, echo pattern, orientation and posterior acoustic features, calcifications, and axillary lymph nodes. The BIRADS category of each lesion was determined according to BIRADS atlas of Ultrasound 2013, guided by the results of clinical data and breast ultrasound findings but blinded to the final pathological diagnosis.

Reference points for us were histopathological analysis of biopsy and surgical samples, fine-needle aspiration cytology, or close follow-up. 


\section{Results}

The study included 51 patients who performed mammography examination with identified breast asymmetries. All patients performed full-field digital mammography, 3D tomosynthesis, and ultrasound.

Their ages ranged from 26 to 72 years with a mean age $50.05 \pm 8.1$ (mean $\pm \mathrm{SD}$ ). Young-aged females came to the mammography department as they were complaining of pain with high-risk family history (two first-degree relatives).

\section{Breast density ACR scoring}

As described and represented in Fig. 1, Breast density ACR scoring was done according to the ACR BIRADS lexicon breast density classification [6], noting that ACR $\mathrm{A}=$ entirely fatty breast, $\mathrm{ACR} \mathrm{B}=$ scattered fibroglandular density, $\mathrm{ACR} C=$ heterogeneously dense breast, and ACR D = extremely dense breast.

Final diagnosis was according to histopathological analysis of biopsy and surgical samples, fine-needle aspiration cytology, or close follow-up. Close follow-up was done for lesions with mostly benign diagnostic imaging criteria, and we did it after 3 months of using US. They were divided into benign and malignant lesions. Benign lesions were 18 (about 31.6\%) while the malignant lesions were 39 (about 68.4\%)

The different pathological entities within the "benign lesions" group are seen in Table 1.

The most common pathological type in malignant lesions was ductal carcinoma in situ (DCIS), invasive ductal carcinoma (IDC), and invasive lobular carcinoma

\section{Distribution of cases according to the ACR BIRAD lexicon breast density classification}

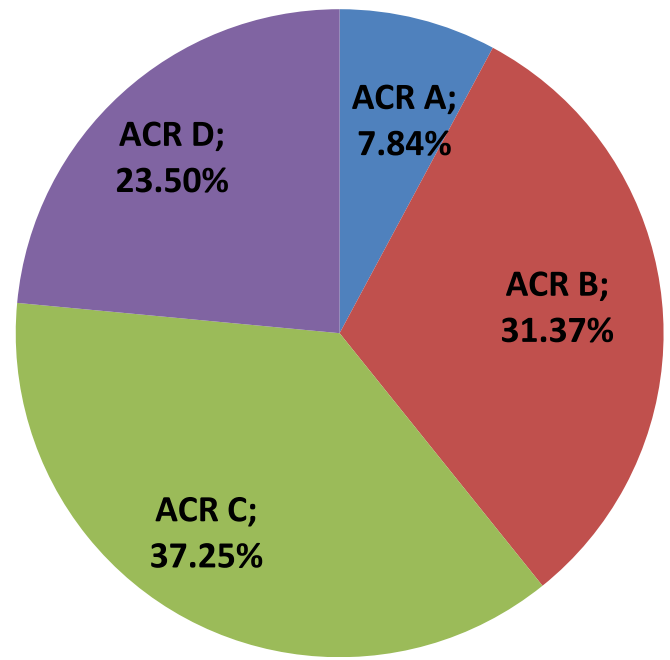

Fig. 1 Distribution of cases according to the ACR BIRADS lexicon breast density classification
Table 1 The distribution of different pathological entities within the benign and malignant lesions

\begin{tabular}{llll}
\hline $\begin{array}{l}\text { Type of } \\
\text { lesion }\end{array}$ & Diagnosis & $\begin{array}{l}\text { Number } \\
\text { of lesions }\end{array}$ & $\begin{array}{l}\text { Percentage } \\
(\%)\end{array}$ \\
\hline Benign & Hamartoma & $1 / 39$ & $2.6 \%$ \\
& Duct ectasia & $1 / 39$ & $2.6 \%$ \\
& Fibroadenoma & $9 / 39$ & $23 \%$ \\
& Intramammary LN & $2 / 39$ & $5.1 \%$ \\
& fibrocystic changes & $7 / 39$ & $17.9 \%$ \\
& Adenosis & $8 / 39$ & $20.5 \%$ \\
& Benign postoperative sequel & $6 / 39$ & $15.4 \%$ \\
& Abscess & $1 / 39$ & $2.6 \%$ \\
& Granulomatous mastitis & $1 / 39$ & $2.6 \%$ \\
& Cluster cysts & $1 / 39$ & $2.6 \%$ \\
& Radial scars & $2 / 39$ & $5.1 \%$ \\
Malignant & IDC & $13 / 18$ & $(72 \%)$ \\
& DCIS and IDC & $3 / 18$ & $(17 \%)$ \\
& ILC & $2 / 18$ & $(11 \%)$ \\
\hline
\end{tabular}

(ILC). The different pathological entities within the "malignant lesions" group are seen in Table 1.

\section{Types of asymmetries by mammography}

Patients were divided into four groups according to the type of asymmetry: simple asymmetry, focal asymmetry, global asymmetry, and developing asymmetry, as shown in Fig. 2.

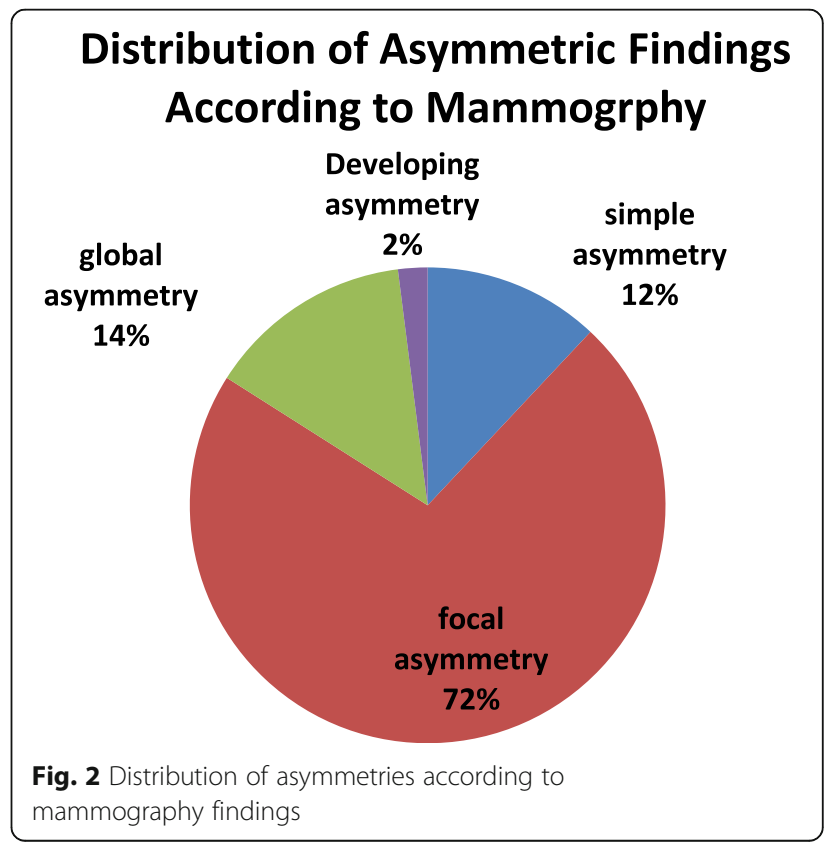


Table 2 Distribution of benign and malignant lesions within the different types of asymmetries

\begin{tabular}{lllll}
\hline & Simple asymmetry & Focal asymmetry & Global asymmetry & Developing asymmetry \\
\hline Number of benign lesion & $4 / 7(57 \%)$ & $32 / 42(76 \%)$ & $3 / 8(37.5 \%)$ & $1 / 1(100 \%)$ \\
Number of malignant lesion & $3 / 7(43 \%)$ & $10 / 42(24 \%)$ & $5 / 8(62.5 \%)$ & 0 \\
Total & $7(100 \%)$ & $42(100 \%)$ & $8(100 \%)$ & $1(100 \%)$
\end{tabular}

The distribution of benign and malignant lesions within the different types of asymmetries is shown in Table 2.

The BIRADS Categories by finding include mammography, 3D digital breast tomosynthesis, ultrasonography, combined digital breast mammography and 3D tomosynthesis, and finally combined digital breast mammography, 3D tomosynthesis, and breast ultrasonography, all of which were described in Tables $3,4,5,6$, and 7 , respectively.

Figures 3, 4, 5, 6, and 7 described the correlation between the diagnostic imaging findings. The final diagnosis mentions how many cases per each (by histopathological analysis of biopsy and surgical samples, fine-needle aspiration cytology, or close follow-up). They describe the diagnostic imaging findings in mammography, 3D digital breast tomosynthesis, ultrasonography, (combined digital breast mammography and 3D tomosynthesis), and finally (combined digital breast mammography, 3D tomosynthesis, and breast ultrasonography).

Based on the previous results described in Tables 5, 6, and 7 and Figs. 3, 4, 5, 6, and 7, the following statistical results were found: Sensitivity of mammography was $72.22 \%$, the specificity was $71.79 \%$, the positive predictive value was $54.17 \%$, the negative predictive value was $84.85 \%$, and the accuracy was $71.92 \%$.

While the sensitivity of tomosynthesis is $83.33 \%$, the specificity was $89.74 \%$, the positive predictive value was $78.95 \%$, the negative predictive value was $92.11 \%$, and the accuracy was $87.71 \%$.

As for breast ultrasound, the sensitivity was $100.00 \%$, the specificity was $92.31 \%$, the positive predictive value was $85.71 \%$, the negative predictive value was $100.00 \%$, and the accuracy was $94.73 \%$.

In combined digital breast mammography and 3D tomosynthesis, it was found that the sensitivity was $83.33 \%$, the specificity was $87.18 \%$, the positive predictive value was $75.00 \%$, the negative predictive value was $91.89 \%$, and the accuracy was $85.96 \%$.

Table 3 The BI-RADS categories by mammography

\begin{tabular}{lll}
\hline BIRADS & Number & Percentage \\
\hline BIRADS & $211 / 57$ & $19 \%$ \\
BIRADS 3 & $22 / 57$ & $39 \%$ \\
BIRADS 4 & $24 / 57$ & $42 \%$ \\
Total & 57 & $100 \%$ \\
\hline
\end{tabular}

In combined digital breast mammography, 3D tomosynthesis, and breast ultrasound, sensitivity was $100.00 \%$, the specificity was $92.31 \%$, the positive predictive value was $85.71 \%$, the negative predictive value was $100.00 \%$, and the accuracy was $94.73 \%$.

We selected some cases to be presented as seen in Figs. 8, 9, 10, and 11 .

\section{Discussion}

The term asymmetric breast tissue refers to a greater volume or density of breast tissue in one breast than in the corresponding area in the contralateral breast. Although asymmetry is often a normal finding, additional evaluation may sometimes be required. Asymmetry may be secondary to removal of tissue or to lack of development or more prominent parenchyma in one breast [2].

For more accurate work-up and diagnosis of soft tissue findings at mammography, the American College of Radiology (ACR) Breast Imaging Reporting and Data System (BIRADS) lexicon provides definitions for four different types of asymmetric breast findings: (1) singleview asymmetry, (2) global asymmetry, (3) focal asymmetry, and (4) developing asymmetry [3].

The aim of this study was to evaluate the diagnostic value of digital breast tomosynthesis (DBT) versus US in the evaluation of breast asymmetries regarding the sensitivity, specificity, positive predictive value, negative predictive value, and accuracy.

The study included 51 patients with 57 breast asymmetries seen on full-field digital mammography (FFDM). Their ages ranged from 26 to 72 years, mean age (50.05 $\pm 8.1 \mathrm{SD})$.

All patients underwent FFDM, DBT, and breast ultrasound.

In this study, $4 / 51$ (7.84\%) cases were assigned an ACR score of "A," 16/51 (31.37\%) cases were assigned an ACR score of "B," 19/51 (37.25\%) cases were assigned an ACR score of "C," 12/51 (23.5\%) were assigned an ACR score of "D."

Table 4 showing the BI-RADS categories by Tomosynthesis

\begin{tabular}{lll}
\hline BIRADS & Number & Percentage \\
\hline BIRADS 1 & $7 / 57$ & $12 \%$ \\
BIRADS 2 & $19 / 57$ & $33 \%$ \\
BIRADS 3 & $12 / 57$ & $21 \%$ \\
BIRADS 4 & $9 / 57$ & $16 \%$ \\
BIRADS 5 & $10 / 57$ & $18 \%$ \\
\hline
\end{tabular}


Table 5 showing the BI-RADS categories by breast ultrasound

\begin{tabular}{lll}
\hline BIRADS & Number & Percentage \\
\hline BIRADS 1 & $8 / 57$ & $14 \%$ \\
BIRADS 2 & $21 / 57$ & $37 \%$ \\
BIRADS 3 & $7 / 57$ & $12 \%$ \\
BIRADS 4 & $4 / 57$ & $7 \%$ \\
BIRADS 5 & $17 / 57$ & $30 \%$ \\
Total & 57 & $100 \%$ \\
\hline
\end{tabular}

Regarding asymmetric findings in this study, 7/57 (12\%) lesions presented as simple asymmetry, 41/57 (72\%) lesions presented as focal asymmetry, 8/57 (14\%) lesions presented as global asymmetry, and 1/57 (2\%) lesions presented as developing asymmetry.

The majority of the breast asymmetries in the current study were secondary to benign lesions 39/57 (68.4\%).

Our results are in accordance to that of Price et al. [3] who found that the majority of breast asymmetries prove to be benign pathologic entities secondary to focal fibrosis, fibrocystic change, fibroadenoma, and pseudoangiomatous stromal hyperplasia (PASH).

Upon correlating with final diagnosis by either by histopathological analysis of biopsy samples, fine-needle aspiration cytology, or close follow-up, there were 39/57 (68.4\%) benign lesions and $18 / 57$ (31.6\%) malignant lesions.

\section{Mammography results}

Mammography BIRADS category was given for each lesion according to the BIRADS mammography morphology descriptors; $33 / 57$ (58\%) lesions were considered benign (BIRADS 1, 2, and 3), while 24/57 (42\%) lesions were considered malignant (BIRADS 4 and 5).

After revising the pathology results, 13/18 (72.22\%) lesions were true positives, $11 / 39$ (28.21\%) lesions were false positive, $5 / 18$ (27.77\%) lesions were false negatives, and $28 / 39$ (71.79\%) lesions were true negatives (as case in Fig. 11).

In this study, the false-positive results are due to overlapping of fibro-glandular tissue, increase breast density, or irregular margins of a benign lesion. The false-

Table 6 The BI-RADS categories by combined digital breast mammography and 3D Tomosynthesis

\begin{tabular}{lll}
\hline BIRADS & Number & Percentage \\
\hline BIRADS 1 & $7 / 57$ & $12 \%$ \\
BIRADS 2 & $19 / 57$ & $33 \%$ \\
BIRADS 3 & $11 / 57$ & $19 \%$ \\
BIRADS 4 & $10 / 57$ & $18 \%$ \\
BIRADS 5 & $10 / 57$ & $18 \%$ \\
Total & 57 & $100 \%$ \\
\hline
\end{tabular}

Table 7 showing the BI-RADS categories by combined digital breast Mammography, 3D Tomosynthesis \& Breast ultrasound

\begin{tabular}{lll}
\hline BIRADS & Number & Percentage \\
\hline BIRADS 1 & $8 / 57$ & $14 \%$ \\
BIRADS 2 & $22 / 57$ & $38 \%$ \\
BIRADS 3 & $6 / 57$ & $11 \%$ \\
BIRADS 4 & $4 / 57$ & $7 \%$ \\
BIRADS 5 & $17 / 57$ & $30 \%$ \\
Total & 57 & $100 \%$ \\
\hline
\end{tabular}

negative results are due to obscured malignant lesions in ACR C and D heterogeneous dense breast.

Pinto et al. [7] who compared between FFDM and DBT in 46 cases of breast asymmetries found that 19 (39.58\%) did not change with DBT and 27 (60.41\%) attenuated or disappeared. Therefore, they concluded that DBT can be useful in the differentiation between the asymmetries and the overlapping tissues, allowing a decrease in the number of unnecessary biopsies in these cases.

In this study, mammography had a sensitivity of $72.22 \%$, a specificity of $71.79 \%$, a positive predictive value of $54.17 \%$, and a negative predictive value of 84.85 $\%$.

Bae et al. (8) stated that mammography is the only screening test that has been proved to reduce breast cancer mortality. However, mammography has pitfalls as a breast imaging modality, with an overall sensitivity of $75-85 \%$, which can decrease to $30-50 \%$ in women with dense breast tissue.

\section{D digital tomosynthesis results}

A BIRADS category was given to lesions identified on 3D digital tomosynthesis according to the Mammography BIRADS Lexicon and accordingly 38/57 (67\%) lesions were considered benign (BIRADS 1, 2, and 3), while 19/57 (33\%) lesions were considered malignant.

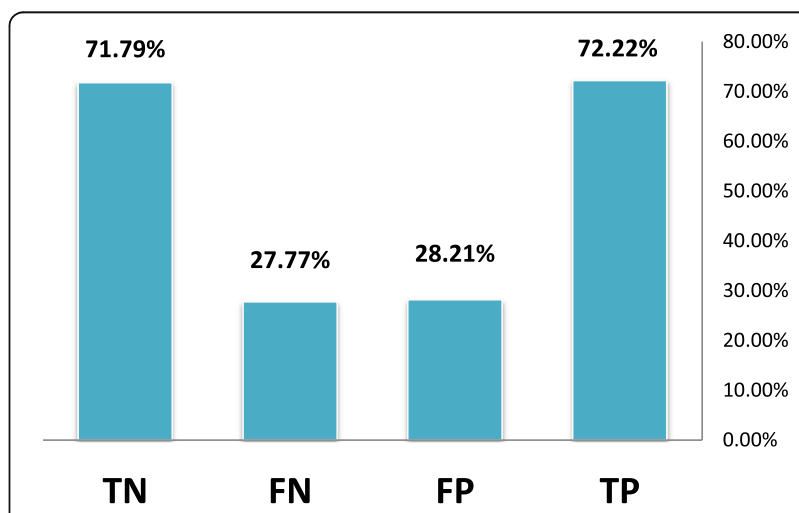

Fig. 3 Association between mammography findings and final diagnoses in the studied group 


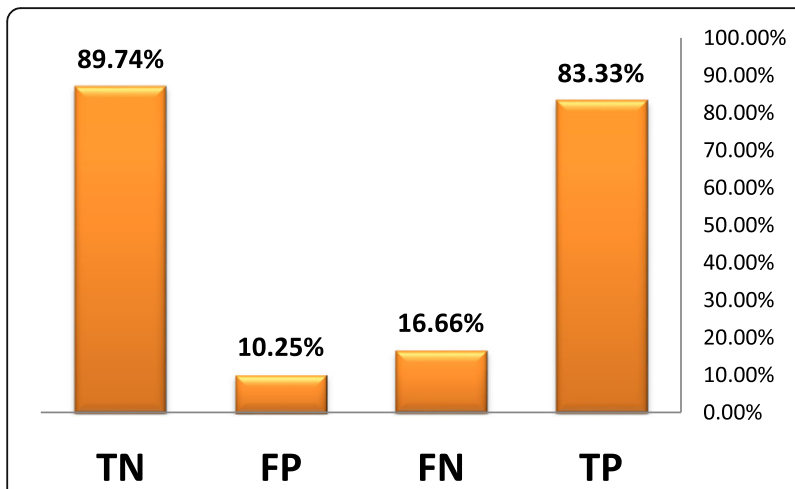

Fig. 4 Association between tomosynthesis findings and final diagnoses in the studied group

After revising the pathology results, 15/18 (83.33\%) lesions were true positives, $4 / 39$ (10.25\%) lesions were false positive, $3 / 18(16.66 \%)$ lesions were false negatives, and 35/39 (89.74\%) lesions were true negatives.

The false-positive results are less (4 instead of 11 cases) when compared to digital mammography. Tomosynthesis overcame the tissue overlap in focal asymmetries and was able to verify if there is an underlying mass or is it only overlapping fibro-glandular tissue. The falsepositive results were due to dense breast or irregular margin of the lesions.

The false-negative results were diffuse subtle infiltration in two cases with diffuse edema and one case with a deeply seated lesion not included in the mammography film view.

Durand et al. [8] found that the use of tomosynthesis $(2 \mathrm{D}+3 \mathrm{D})$ compared with conventional mammography (2D) is associated with a lower recall rate of screening mammography, most often for asymmetries.

Nam et al. [9] stated that lesion characterization of digital breast tomosynthesis (DBT) was more specific than that of full-field digital mammography (FFDM), and focal asymmetry or mass terminology was more frequently used in DBT (53.6\%) than in FFDM (17.1\%),

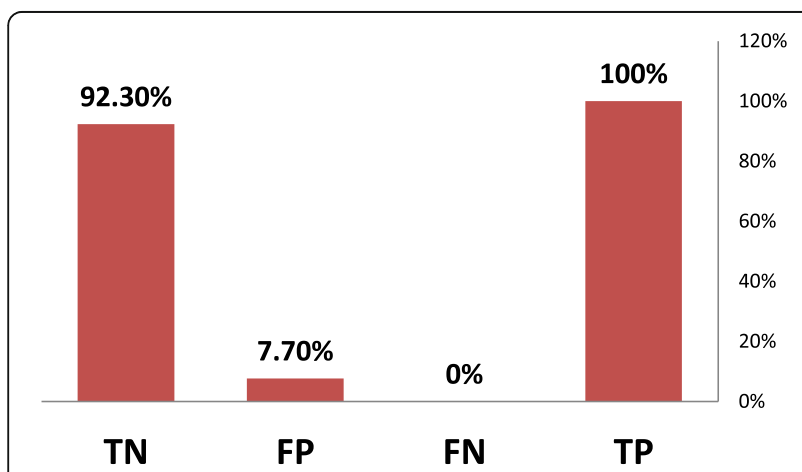

Fig. 5 Association between ultrasound findings and final diagnosis in the studied group

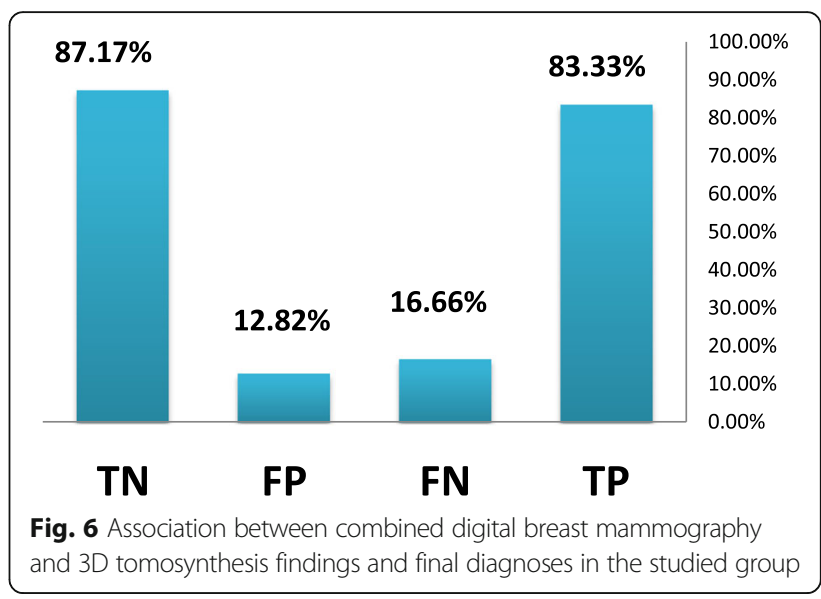

whereas asymmetry terminology was less frequently used in DBT (12.2\%) than in FFDM (31.7\%) by the informed radiologists.

In this study, tomosynthesis had a sensitivity of 83.33 $\%$, a specificity of $89.74 \%$, a positive predictive value of $78.95 \%$, and a negative predictive value of $92.11 \%$.

Peppard et al. [10] stated that DBT is often useful for evaluation of a focal asymmetry. It can be used to confirm and characterize a finding as a true asymmetry, dismiss it as a superimposition or reclassify it as a mass.

In a study by El Maadawy et al. [11] who used DBT for evaluation of focal mammographic asymmetry, DBT showed higher sensitivity (93.8\%) for lesion visualization and differentiation of true lesions from summation artifacts than did spot compression imaging (50.2\%).

\section{Breast ultrasound results}

Ultrasound BIRADS category was given for each lesion according to the BIRADS mammography morphology descriptors; 36/57 (63\%) lesions were considered benign (BIRADS 1, 2, and 3), while 21/57 (37\%) lesions were considered malignant.

After revising the pathology results, 18/18 (100.00\%) lesions were true positives (as the case in Fig. 8), 3/39

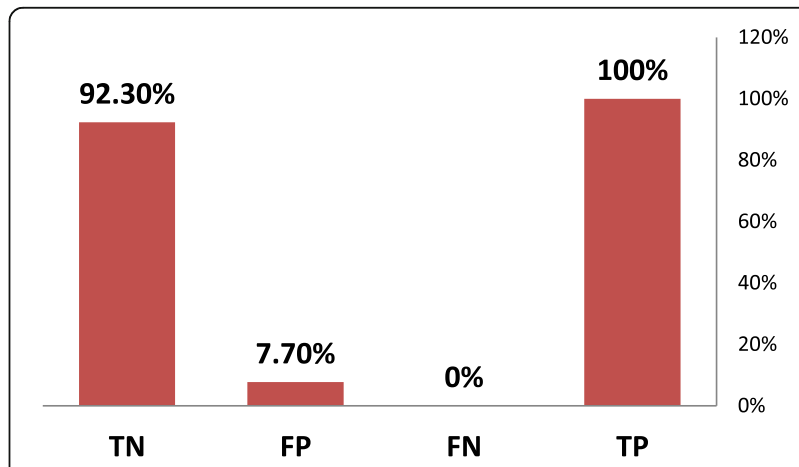

Fig. 7 Association between combined digital breast mammography, tomosynthesis and breast ultrasound findings, and final diagnoses in the studied group 

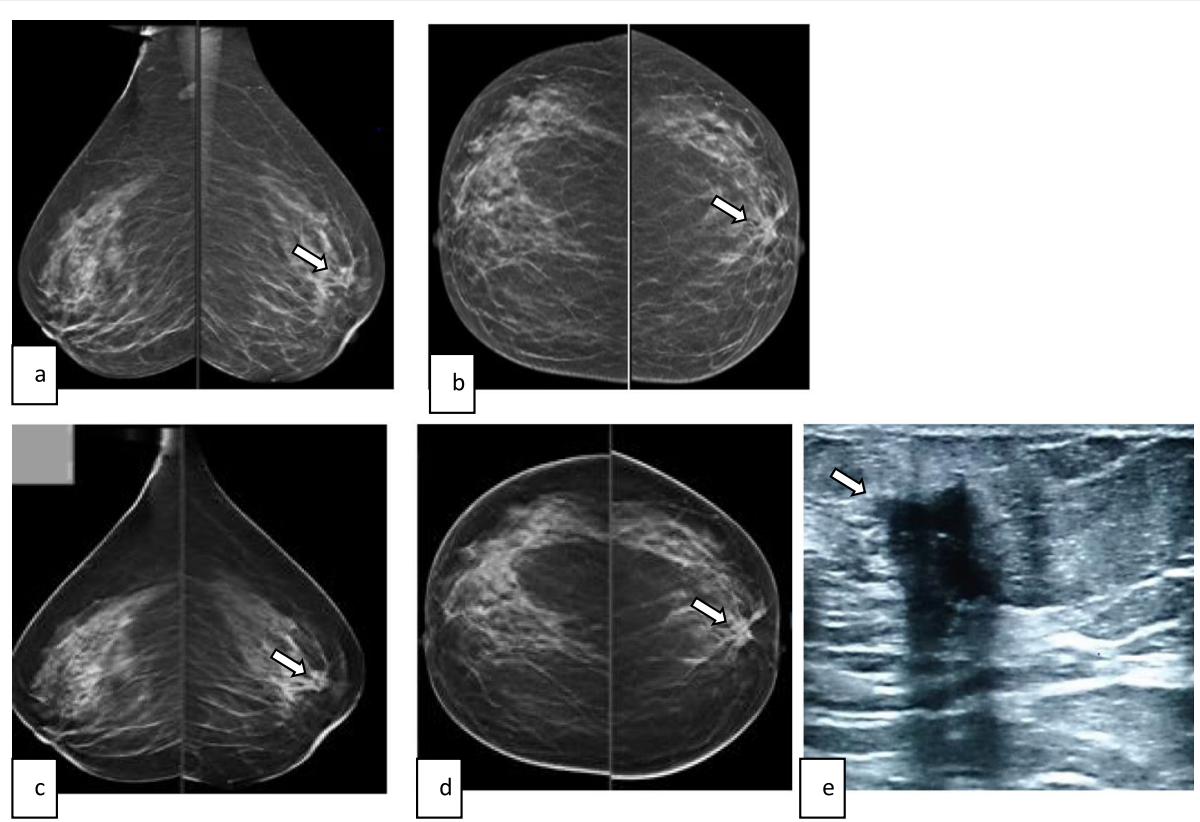

Fig. 8 (Case no. 1) A 52-year-old patient presented by mastalgia and palpable mass in the left breast. Mammography revealed breast density: ACR B and left retro-areolar focal asymmetry assigned as (BIRADS 4) (a, b). 3D tomosynthesis revealed: left retro-areolar speculated mass lesion is seen (BIRADS5) (c, d). Breast ultrasound revealed: an irregular speculated hypoechoic mass lesion with posterior shadowing (BIRADS 5) (e). Final diagnosis: invasive duct carcinoma
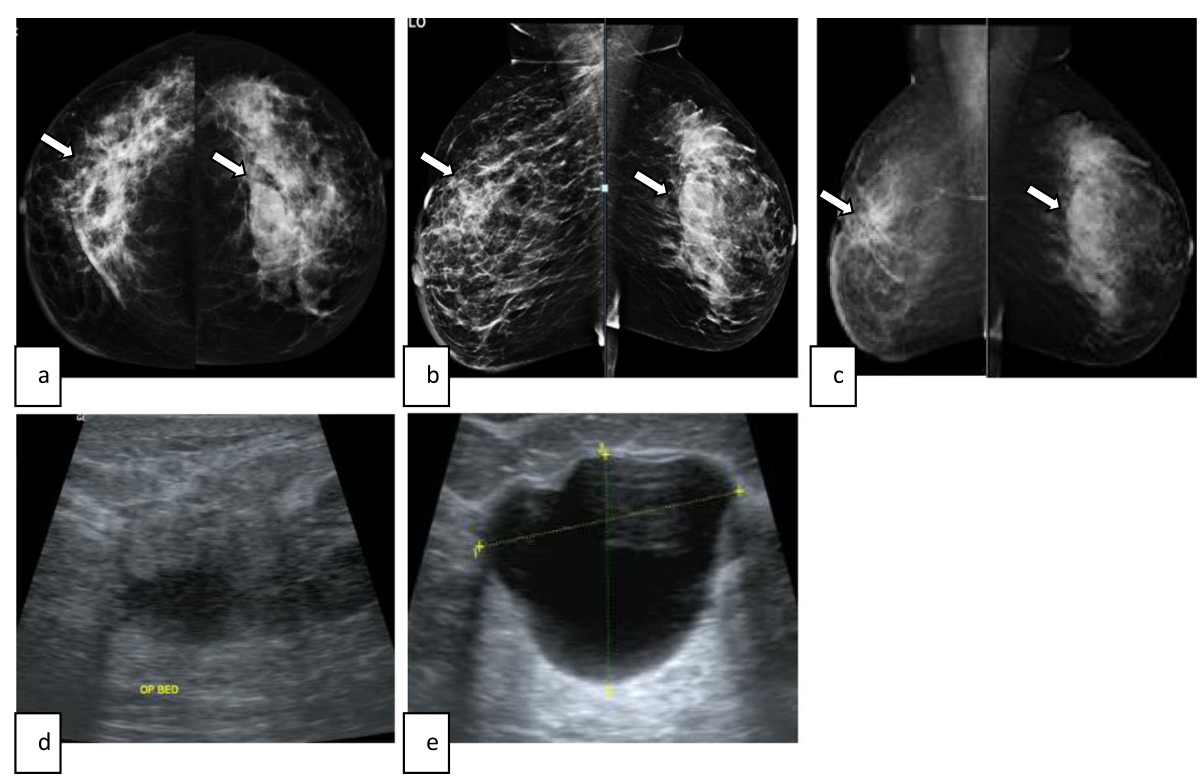

Fig. 9 (Case no. 2) A 58-year-old patient with history of right breast conservative surgery and left mastalgia. Mammography revealed breast density: ACR B, right breast: single-view asymmetry (BIRADS 3) and left breast: global asymmetry (BIRADS 4) (a and b show CC and MLO views, respectively). 3D tomosynthesis was inconclusive in both sides (c MLO view). Breast ultrasound revealed: right breast: operative bed architecture distortion (BIRADS 2) (d) and left breast: well-defined thin wall hypoechoic cyst with posterior enhancement (BIRADS 2) (e). Final diagnosis: right breast postoperative changes and left breast fibrocystic changes 


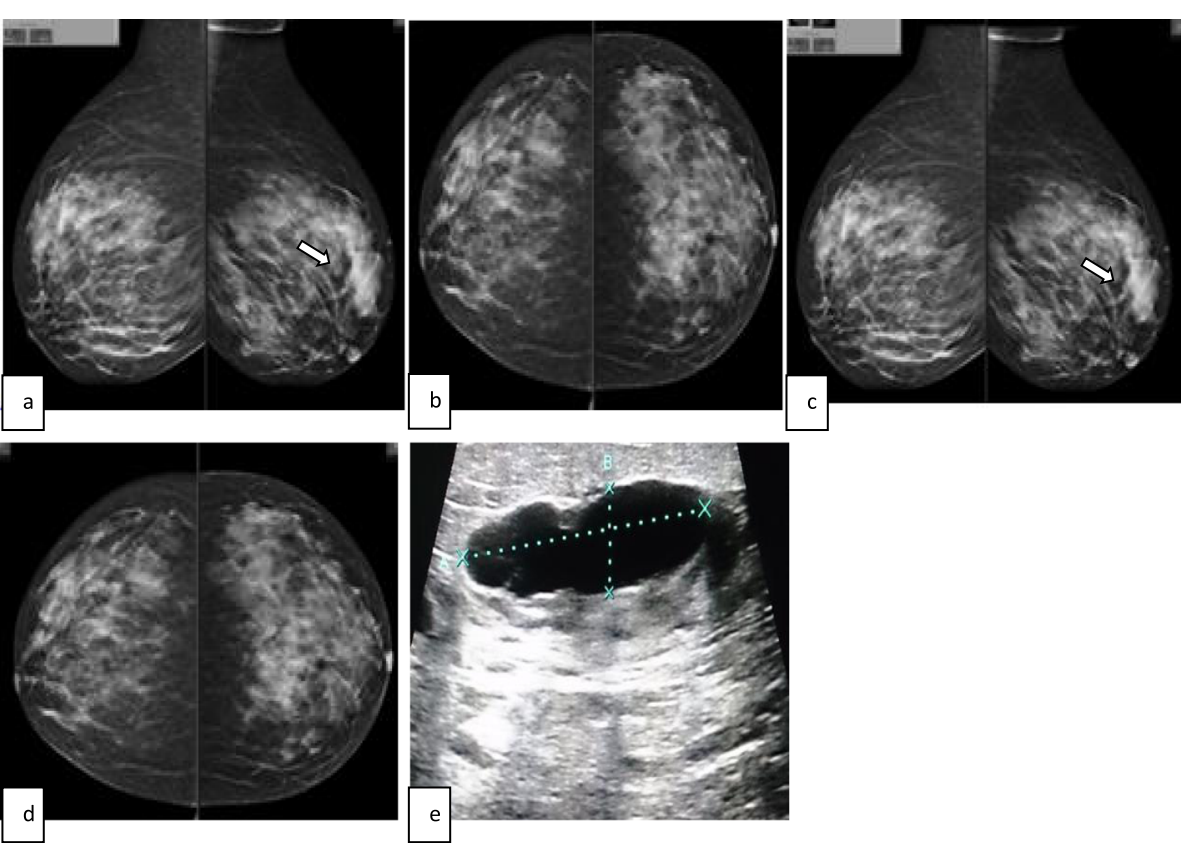

Fig. 10 (Case no. 3) A 48-year-old patient came for regular screening. Mammography revealed breast density: ACR C. and left upper outer quadrant focal asymmetry (BIRADS3) (a, b). 3D tomosynthesis revealed: left upper outer quadrant focal asymmetry (BIRADS 3), tomosynthesis did not add to the mammography $(\mathbf{c}, \mathbf{d})$. Breast ultrasound revealed: multiple well-defined variable-sized thin wall anechoic cysts with posterior enhancement, the largest measuring $(3.5 \times 1 \mathrm{~cm})($ BIRADS 2) $(\mathbf{e})$. Final diagnosis: fibrocystic changes
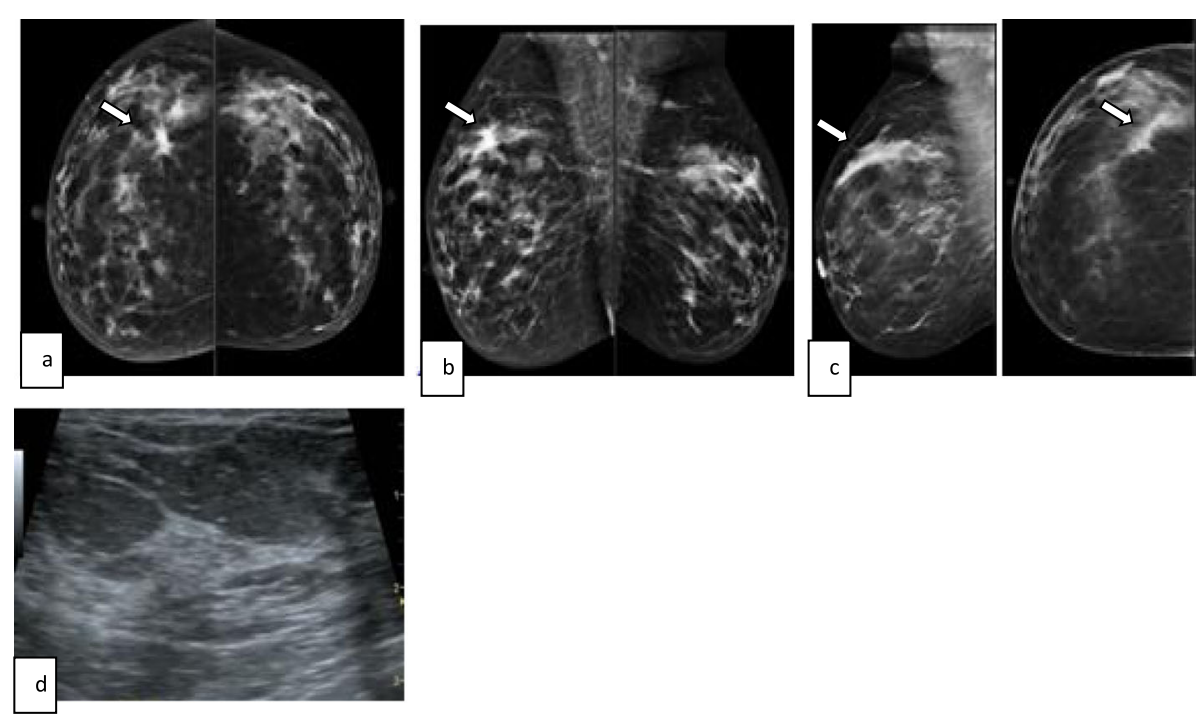

Fig. 11 (Case no. 4) A 26-year-old patient. She came for mastalgia with high-risk positive family history (two first-degree relatives). Mammography revealed breast density: ACR C. Right upper outer quadrant focal asymmetry (BIRADS4) (a, b). 3D tomosynthesis revealed: right upper outer quadrant normal parenchymal tissue (BIRADS 1) (c). Breast ultrasound revealed hyperehoic parenchyma with mottling appearance denoting adenosis (BIRADS 1) (d). Final diagnosis: breast adenosis 
(7.7\%) lesions were false positive, we had no falsenegative results, and 36/39 (92.30\%) lesions were true negatives. The false-positive results included radial scar, extensive postoperative changes, and granulomatous mastitis). Those lesions are false positive as they had the same morphologic criteria of malignant mass lesion.

Bae et al. [12] stated that in the American College of Radiology Imaging Network (ACRIN) 6666 study, screening US had a sensitivity of $76 \%$ and a specificity of $84 \%$.

In this study, ultrasound had a sensitivity of $100.00 \%$, a specificity of $92.31 \%$, a positive predictive value of $85.71 \%$, and a negative predictive value of $100.00 \%$.

Breast ultrasound in our study showed the highest sensitivity.

Song et al. [13] stated that lesion characteristics (e.g., isoechogenicity, deeply located lesions in large breasts, subtle features) and technical failures have previously been considered reasons for false-negative diagnoses at breast US.

Breast ultrasound proved to be valuable in the characterization of the asymmetric lesions and in further evaluation of abnormalities identified on other breast imaging modalities. It provides information on the internal matrix, shape, margins, and vascular patterns of mammographic asymmetric breast findings that help to differentiate benign from malignant masses with a fairly high degree of certainty. Ultrasound is the preferred initial imaging technique for evaluating symptomatic breast lesions in women younger than 30 years [4].

\section{Combined digital mammography and 3D tomosynthesis findings}

Combined digital mammography and 3D tomosynthesis BIRADS category was given for each lesion according to the BIRADS mammography morphology descriptors; 37/ 57 (65\%) lesions were considered benign (BIRADS 1, 2, and 3), while 20/57 (35\%) lesions were considered malignant.

After revising the pathology results 15/18 (83.33 \%) lesions were true positives, $5 / 39$ (12.82\%) lesions were false positive, $3 / 18$ (16.66) lesion was false negative, and $34 / 39$ (87.17\%) lesions were true negatives.

The false-positive results are less (became 5 instead of 11 case) when compared to digital mammography alone as tomosynthesis overcame the tissue overlap in focal asymmetries.

So, combined digital breast mammography and 3D tomosynthesis had a sensitivity of $88.33 \%$, a specificity of $87.18 \%$, a positive predictive value of $75.00 \%$, and a negative predictive value of $91.89 \%$.

Many researchers have investigated the potential role of DBT in both screening and diagnostic settings. Improvements in sensitivity and specificity are expected after adding DBT to conventional mammography because DBT eliminates overlapping tissues, and lesion margins can be more readily assessed, which may reduce the need for extra views as results of Kim et al (4).

\section{Combined digital mammography, 3D tomosynthesis, and ultrasound findings}

Combined digital mammography, 3D tomosynthesis, and ultrasound BIRADS category was given for each lesion according to the BIRADS mammography morphology descriptors; 36/57 (63\%) lesions were considered benign (BIRADS 1, 2, and 3) while 21/57 (37\%) lesions were considered malignant.

After revising the pathology results, 18 (100.00 \%) lesions were true positives, $3(7.7 \%)$ lesions were false positive, $0(0 \%)$ lesions was false negative, and 36 $(92.30 \%)$ lesions were true negatives.

Kim et al. [4] found that previous prospective clinical studies have demonstrated that appropriate use of US as an adjunct to mammography improves sensitivity and specificity of breast cancer diagnoses, particularly in women with dense breasts and in younger women.

In this study, combined digital mammography, 3D tomosynthesis, and ultrasound had a sensitivity of $100.00 \%$, a specificity of $92.31 \%$, a positive predictive value of $85.71 \%$, and a negative predictive value of $100.00 \%$.

However, some points made were usage of 3D (DBT) is limited, like relatively higher dose of radiation, higher cost, and less availability than FFDM. Considering this study, decreased number of patients may make the results a matter of discussion.

\section{Conclusion}

From this study, we recommend that 3D digital tomosynthesis should be coupled with FFDM in the screening context to decrease the recall rate as it resolves the problem of tissue overlap in dense breast.

In the diagnostic context, ultrasound proved to be more accurate relative to tomosynthesis in characterization of lesion underlying breast asymmetries.

\section{Abbreviations \\ DBT: Digital breast tomosynthesis; FFDM: Full-field digital mammography; US: Ultrasound; MLO: Mediolateral-oblique; CC: Cranial-caudal; BIRADS: Breast Imaging Reporting and Data System; TP/FP: True positive/false positive; TN/ FN: True negative/false negative; IDC: Invasive ductal carcinoma; DCIS: Ductal carcinoma in situ; ILC: Invasive lobular carcinoma}

\section{Acknowledgements}

We acknowledge the patients whose accept using their data and the radiology staff help us in data collection

\section{Authors' contributions}

OM collected cases and wrote the manuscript. SM edited the images and text. GG contributed to the manuscript and its revision. EN collected cases. MG contributed to the manuscript and its revision. All authors read and approved the final manuscript. 


\section{Funding}

This study had no funding from any resource.

\section{Availability of data and materials}

The datasets used and/or analyzed during the current study are available from the corresponding author on reasonable request. You could contact with Dr. Omnia Mokhtar.

\section{Ethics approval and consent to participate}

All study procedures were conducted in accordance with the Declaration of Helsinki and were approved by the National Cancer Institute ethics committee/Cairo University, Egypt. The Ethics committee reference number in the "Ethics Approval and Consent to Participate" is (201920003.2P). All patients included in this study gave us verbal consent to participate and were all adults, so no parental consent was taken

\section{Consent for publication}

All patients included in this research gave informed consent to publish the data contained within this study.

\section{Competing interests}

The authors declare that they have no competing interests.

\section{Author details}

${ }^{1}$ Department of Diagnostic and Interventional Radiology, National Cancer Institute Cairo University, Cairo, Egypt. ²Department of Diagnostic Radiology, Beni-suef University, Beni-suef, Egypt. ${ }^{3}$ Radiology Department, Faculty of Medicine, October 6 University, Cairo, Egypt. ${ }^{4}$ Ministry of Health, Cairo, Egypt. ${ }^{5}$ Department of Diagnostic and Interventional Radiology, National Liver Institute, Menofyia University, Al Minufya, Egypt. ${ }^{6} \mathrm{Al}-\mathrm{Haram}$ Al-, Giza, Egypt.

Received: 30 December 2019 Accepted: 5 May 2020

Published online: 23 June 2020

\section{References}

1. Chesebro AL, Winkler NS, Birdwell RL, Giess CS (2016) Developing asymmetries at mammography: a multi-modality approach to assessment and management. RadioGraphics 36:322-334

2. AbdulWahid H. Khalel E. and Alwan N. (2019). Mammographic, ultrasonographic and pathologic correlations of focal asymmetric breast densities among a sample of Iraqi women. Journal of Contemporary Medical Sciences. Vol 5 No 3 : May-June

3. Price ER, Joe BN, Sickles EA (2015) The developing asymmetry: revisiting a perceptual and diagnostic: radiology. Radiology March. 274(3):642-651

4. Kim SA, Chang JM, Cho N, Yi A, Kyung Moon W (2015) Characterization of breast lesions: comparison of digital breast tomosynthesis and ultrasonography. Korean J Radiol. 16(2):229-238

5. Li X, Qin G, He Q, Sun L, Zeng H, He Z, Chen W, Zhen X, Zhou L (2019) Digital breast tomosynthesis versus digital mammography: integration of image modalities enhances deep learning-based breast mass classification. European Radiology volume 30:778-788

6. American College of Radiology, BI-RADS ${ }^{\circledR}$ ATLAS - MAMMOGRAPHY (2013) :124. https://www.acr.org/-/media/ACR/Files/RADS/BI-RADS/MammographyReporting.pdf

7. Pinto L, Sondermann V, Caldoncelli V, Pereira P, Pasqualette H. (2015) Asymmetries: comparison between full field digital mammography and digital breast tomosyntesis. SIS Journal is the Electronic Journal of the Senologic International Society, the World Society of Breast Diseases. :16888170.

8. Durand MA, Haas BM, Yao X, Geisel JL, Raghu M, Hooley RJ, Horvath L, Philpotts LE (2015) Early clinical experience with digital breast tomosynthesis for screening mammography. Radiology. 274(1):85-92

9. Nam KJ, Han BK, Ko ES, Choi JS, Ko EY, Jeong DW, Choo KS (2015) Comparison of full-field digital mammography and digital breast tomosynthesis in ultrasonography-detected breast cancers. the. Breast. 24(5): 640-655

10. Peppard HR, Nicholson BE, Rochman CM, Merchant JK, Mayo RC III, Harvey JA (2015) Digital breast tomosynthesis in the diagnostic setting: indications and clinical applications. RadioGraphics. 35(4):975-990

11. ElMaadawy MM, Seely JM, Doherty G, Lad SV. (2012) Digital breast tomosynthesis in the evaluation of focal mammographic asymmetry: do you still need coned compression views? [abstr]. In: Radiological Society of North America Scientific Assembly and Annual Meeting Program. Oak Brook, III: Radiological Society of North America. 158.

12. Bae MS, Moon WK, Chang JM, Koo HR, Kim WH, Cho N, Yi A, Yun BL, Lee SH, Kim MY, Ryu EB, Seo M (2014) Breast cancer detected with screening US: reasons for nondetection at mammography. Radiology. 270(2):369-377

13. Song SE, Cho N, Chu A, Shin SU, Yi A, Lee SH, Kim WH, Bae MS, Moon WK (2015) Undiagnosed breast cancer: features at supplemental screening US. Radiology. 277(2):372-380

\section{Publisher's Note}

Springer Nature remains neutral with regard to jurisdictional claims in published maps and institutional affiliations.

\section{Submit your manuscript to a SpringerOpen ${ }^{\circ}$ journal and benefit from:}

- Convenient online submission

- Rigorous peer review

- Open access: articles freely available online

High visibility within the field

- Retaining the copyright to your article

Submit your next manuscript at $\boldsymbol{\nabla}$ springeropen.com 